\title{
Qualidade de vida de atletas Brasileiros, Canadenses e Norte-Americanos
}

\section{Quality of life in Brazilian, Canadian and North American athletes}

\author{
Érico Felden Pereira \\ Universidade do Estado de Santa Catarina - UDESC - Florianópolis - Santa Catarina - Brasil \\ ericofelden@gmail.com \\ Clarissa Stefani Teixeira \\ Sociedade Educacional de Santa Catarina - SOCIESC - Florianópolis - Santa Catarina - Brasil \\ clastefani@gmail.com \\ Luiz Fernando Cuozzo Lemos \\ Universidade Federal do Rio Grande do Sul - UFRGS - Porto Alegre - Rio Grande do Sul - Brasil \\ luizcanoagem@yahoo.com.br \\ Fernanda Tolentino de Souza Bleyer \\ Universidade do Estado de Santa Catarina - UDESC - Florianópolis - Santa Catarina - Brasil \\ fernanda-ts@uol.com.br \\ Douglas Filipin \\ Universidade do Estado de Santa Catarina - UDESC - Florianópolis - Santa Catarina - Brasil \\ fillipin_do@yahoo.com.br \\ Cleber Fernando Rebelatto \\ Universidade do Estado de Santa Catarina - UDESC - Florianópolis - Santa Catarina - Brasil \\ cleberfrebelatto@yahoo.com.br
}

\section{RESUMO}

OBJETIVO: Comparar a qualidade de vida de 19 canoístas e remadores brasileiros, canadenses e norte-americanos nos jogos Pan-americanos de 2007.

MÉTODO: Os atletas responderam um questionário contendo perguntas referentes aos dados pessoais e à qualidade de vida por meio Whogol-bref, esse validado em diferentes culturas e nacionalidades. Foram convidados a participar do estudo todos os atletas de canoagem e remo dos três países. 19 atletas fizeram parte da amostra que foi composta por 6 brasileiros, 4 canadenses e 9 norte-americanos dos quais 6 atletas eram de canoagem e 13 de remo.

RESULTADO: $\mathrm{Na}$ análise da qualidade de vida os brasileiros apresentaram escores superiores aos estrangeiros nos domínios físico $(\mathrm{p}=0,002)$; psicológico $(\mathrm{p}=0,016)$ e relações sociais $(\mathrm{p}=0,018)$. $\mathrm{O}$ domínio físico foi o único que apresentou diferenças entre os sexos $(p=0,026)$. Questões relacionadas à autoestima e ao ambiente no lar foram apontadas como as mais positivas pelos brasileiros. Apesar disso, os atletas estrangeiros apresentam percepção mais positiva de segurança física e proteção. $\mathrm{Na}$ análise da capacidade explicativa dos domínios para a qualidade de vida geral, o domínio meio ambiente foi o que apresentou maior poder preditivo $(24,5 \%)$. 
CONCLUSÃO: Identificou-se que as maiores necessidades dos atletas norte-americanos estão associadas aos domínios físico e relações sociais, dos canadenses ao domínio físico e dos brasileiros ao domínio meio ambiente evidenciando diferenças marcantes entre as diferentes nacionalidades.

PALAVRAS-CHAVE: Qualidade de vida. Atletas. Promoção da saúde.

\section{ABSTRACT}

OBJECTIVE: To compare the quality of life of 19 Brazilian, Canadian and American kayakers and oarsmen in the 2007 Pan American Games.

METHOD: The athletes answered a questionnaire containing questions about data and quality of life through Whoqol-bref, this validated in different cultures and nationalities. Were invited to participate in the study all athletes of canoeing and rowing the three countries. All athletes of kayakers and oarsmen were invited. 19 athletes were part of the sample was composed of six Brazilians, four Canadians and nine North Americans athletes. Six of them kayakers and thirteen were oarsmen.

RESULTS: In analyzing the quality of life Brazilians scored over to foreigners in physical $(\mathrm{p}=0.002)$; psychological $(\mathrm{p}=0.016)$ and social relations $(\mathrm{p}=0.018)$. The physical domain was the only one to showed differences between the sexes $(\mathrm{p}=0.026)$. Related to self-esteem and home environment issues were identified as the most positive by Brazilians. Nevertheless, foreign athletes have more positive perceptions of physical safety and security. In the analysis of the explanatory power of the areas for overall quality of life, the environment domain showed the greatest predictive power $(24.5 \%)$.

CONCLUSION: This study identified that the greatest need of North American athletes are associated with physical and social relationships domains, the physical domain of Canadians and Brazilians environmental domain showed significant differences between the different nationalities.

KEYWORDS: Quality of life. Athletes. Health promotion.

\section{Introdução}

A qualidade de vida é considerada como a "[...] percepção do indivíduo de sua posição na vida no contexto da cultura e sistema de valores nos quais vive e em relação aos seus objetivos, expectativas, padrões e preocupações" (WHOQOL GROUP, 1994). Além disso, podem-se destacar três aspectos fundamentais referentes ao constructo qualidade de vida obtidos por meio das discussões de um grupo de experts de diferentes culturas: (1) subjetividade; (2) multidimensionalidade e (3) presença de dimensões positivas, como a mobilidade, e, negativas, como a dor (WHOQOL GROUP, 1994). A qualidade de vida ainda é apontada como uma dimensão mais abrangente que a saúde sendo um aspecto fundamental para se ter uma boa saúde e não somente o inverso (RENWICK; BROWN, 1996).

Embora as práticas de exercícios físicos sejam tidas como promotoras de saúde nas esferas cognitivas e física, elevando a qualidade de vida dos praticantes (CASPERSEN; POWELL; CHRISTENSON, 1985; WARBURTON; NICOL; BREDIN, 2006), o esporte de elite, quando analisado pela literatura especializada, não é mais apontado, a priori, como sinônimo de saúde devido às grandes prevalências de lesões e outros desfechos em saúde que acometem os atletas de elite (MCALLISTER et al., 2001; KNOWLES; MARSHALL; GUSKIEWICZ, 2006; RECHEL; YARD; COMSTOCK, 2008; WALLS et al., 2010). Soma-se a esse quadro o pouco incentivo e patrocínio, como mostra o estudo de Ferreira e Moraes (2012) no qual foi verificado, nos atletas olímpicos brasileiros de natação, que o apoio inicial financeiro provinha de recursos da família. Além disso, volume grande de treinamento muitas vezes em condições inapropriadas para tal, 
também pode ser apontado como fator prejudicial à saúde e à qualidade de vida dos atletas (SILVA et al., 2013).

Estas condições podem levar a lesões, como estudado por Hensel, Perroni e Leal Junior (2008), que identificaram prevalência de $87,50 \%$ de lesões como contraturas e tendinites em atletas da seleção feminina de canoagem, além de uma reincidência de $82,05 \%$ dessas lesões. Em estudo semelhante, Lemos, Teixeira e Mota (2010) identificaram que 45,45\% das atletas de canoagem apresentavam dores lombares. Da mesma forma, no estudo apresentado por Resch e Haász (2009), no qual investigaram distúrbios alimentares em atletas olímpicos, inclusive de canoagem, identificaram que $73,60 \%$ dos atletas possuíam algum sintoma patológico, além da prevalência de $16,70 \%$ de bulimia nervosa, índices muito superiores às médias populacionais.

De forma geral, as lesões provocam algum tipo de adaptação aos praticantes de atividade física. É o que mostra o estudo de Marwan et al. (2011) que fez o acompanhamento de atletas por 12 meses e identificou que, nesse período, em algumas modalidades esportivas, em torno de 80,00\% dos atletas tiveram algum tipo de lesão e, quando questionados sobre o tempo de afastamento das atividades para recuperação de sua última lesão, $42,00 \%$ dos 452 atletas investigados revelaram ter necessitado de 10 ou mais dias para se recuperar e retornar às suas atividades laborais.

Huffman et al. (2008) apontam a carência de estudos relacionando saúde e qualidade de vida dos atletas, mesmo existindo um grande número de pessoas que participam de competições e treinamento exaustivos, inclusive nos anos escolares, como é comum nos Estados Unidos.

Neste contexto, buscou-se comparar a qualidade de vida de 19 canoístas e remadores brasileiros, canadenses e norte-americanos nos jogos Pan-americanos de 2007.

\section{Método}

O presente estudo investigou a qualidade de vida de 19 atletas dos Jogos Panamericanos de 2007, realizados no Rio de Janeiro. Foram convidados a participar do estudo todos os atletas do Brasil, Canadá e Estados Unidos das modalidades de canoagem e de remo, sendo seis brasileiros (um homem e cinco mulheres), quatro canadenses (todas mulheres) e nove norte-americanos (todos homens). A população foi formada por 6 atletas de canoagem e 13 de remo. A média de idade foi de $25,31( \pm 4,21)$ anos.

Os atletas foram convidados a responder um questionário, em seus idiomas de origem, com dados pessoais e ao Whoqol-bref para avaliação da qualidade de vida. O Whoqol-bref é um instrumento criado pela World Health Organization Quality of Life, traduzido e validado para o Brasil por Fleck et al. (2000) e permite a avaliação e comparação da qualidade de vida em diferentes culturas e nacionalidades.

O Whoqol-bref é formado por 26 questões, incluindo os domínios físico, psicológico, social e ambiental, sendo cada domínio formado por facetas específicas, além de 2 questões gerais sobre saúde e qualidade de vida. As questões são apresentadas com uma pontuação em escala Likert no sentido positivo, ou seja, quanto maior for a pontuação mais positiva é a satisfação com o aspecto analisado. As questões relacionadas à dor e ao desconforto, à dependência de tratamento médico e à ocorrência de sentimentos negativos apresentam sentido inverso e sua pontuação é transformada para a comparação entre as questões. A análise dos escores gerais é realizada por meio de sintaxe própria do instrumento. Os domínios e a percepção geral de saúde e qualidade de vida apresentam escores de 0 a 100 também no sentido positivo e ainda não possuem classificação. Para os atletas brasileiros foi utilizada a versão em português (FLECK et al., 2000) e para os atletas canadenses e norte-americanos utilizou-se a versão em inglês (WHOQOL GROUP, 1994).

As diferenças entre as médias foram analisadas por meio do teste de Kruskal-Wallis com post-hoc o teste de Dunn. A capacidade preditiva dos domínios em relação à qualidade de vida geral foi realizada por meio de regressão linear múltipla utilizando a qualidade de vida geral como variável dependente e como variáveis independentes os escores totais dos domínios. O modo de calculo Backward foi selecionado para a regressão linear múltipla e apresentou diferentes possibilidades de arranjos das variáveis independentes. A análise residual do modelo de regressão 
ajustado foi confirmada. Adotou-se, em todas as análises estatísticas, um nível de probabilidade de significância de 5,00\%.

A pesquisa foi aprovada pelo Comitê de Ética e Pesquisa da Universidade Federal de Santa Maria ( $\mathrm{n}^{\circ}$ 0095.0.243.000-07).

\section{Resultados}

As análises de diferenças entre médias dos domínios da qualidade de vida de acordo com a nacionalidade mostraram-se significativas nos domínios físico, psicológico e relações sociais (Tabela 1). Os atletas brasileiros se diferenciaram dos demais, especialmente no domínio físico, apresentando índices superiores de satisfação para as facetas específicas. As diferenças nos domínios psicológico $(\mathrm{p}=0,016)$ e relações sociais $(\mathrm{p}=0,018)$ foram mais marcantes comparando atletas brasileiros e norte-americanos. Não foram observadas correlações significativas entre a idade e os domínios de qualidade de vida.

Tabela 1 Resultados das análises da qualidade vida geral e dos domínios do Whoqol-bref de acordo com a nacionalidade

\begin{tabular}{|c|c|c|c|c|c|c|c|}
\hline \multirow{3}{*}{ Domínios } & \multicolumn{7}{|c|}{ País } \\
\hline & Brasil & \multirow{2}{*}{$\Delta$} & Canadá & \multirow{2}{*}{$\Delta$} & EUA & \multirow{2}{*}{$\Delta$} & \multirow{2}{*}{ p-valor } \\
\hline & $\mathrm{x}(\mathrm{s})$ & & $\mathrm{x}(\mathrm{s})$ & & $\mathrm{x}(\mathrm{s})$ & & \\
\hline Geral & $72,00(5,1)$ & $\mathrm{a}$ & $72,89(7,4)$ & $\mathrm{a}$ & $72,00(6,5)$ & $\mathrm{a}$ & 0,941 \\
\hline Físico & $86,90(10,7)$ & $\mathrm{a}$ & $51,19(9,1)$ & $\mathrm{b}$ & $62,35(10,6)$ & $\mathrm{b}$ & $<0,002$ \\
\hline Psicológico & $79,86(4,9)$ & $\mathrm{a}$ & $72,68(5,6)$ & $\mathrm{a}, \mathrm{b}$ & $66,67(5,9)$ & $\mathrm{b}$ & 0,016 \\
\hline Relações sociais & $81,25(5,1)$ & $\mathrm{a}$ & $69,44(13,8)$ & $a, b$ & $54,16(17,3)$ & $\mathrm{b}$ & 0,018 \\
\hline Meio ambiente & $69,79(10,8)$ & $\mathrm{a}$ & $71,18(11,8)$ & $\mathrm{a}$ & $68,75(12,7)$ & $\mathrm{a}$ & 0,895 \\
\hline
\end{tabular}

A comparação entre os sexos (Gráfico 1) mostrou diferenças significativas apenas no domínio físico $(\mathrm{p}=0,026)$.

Gráfico 1 Valores médios das análises da qualidade de vida de acordo com o gênero

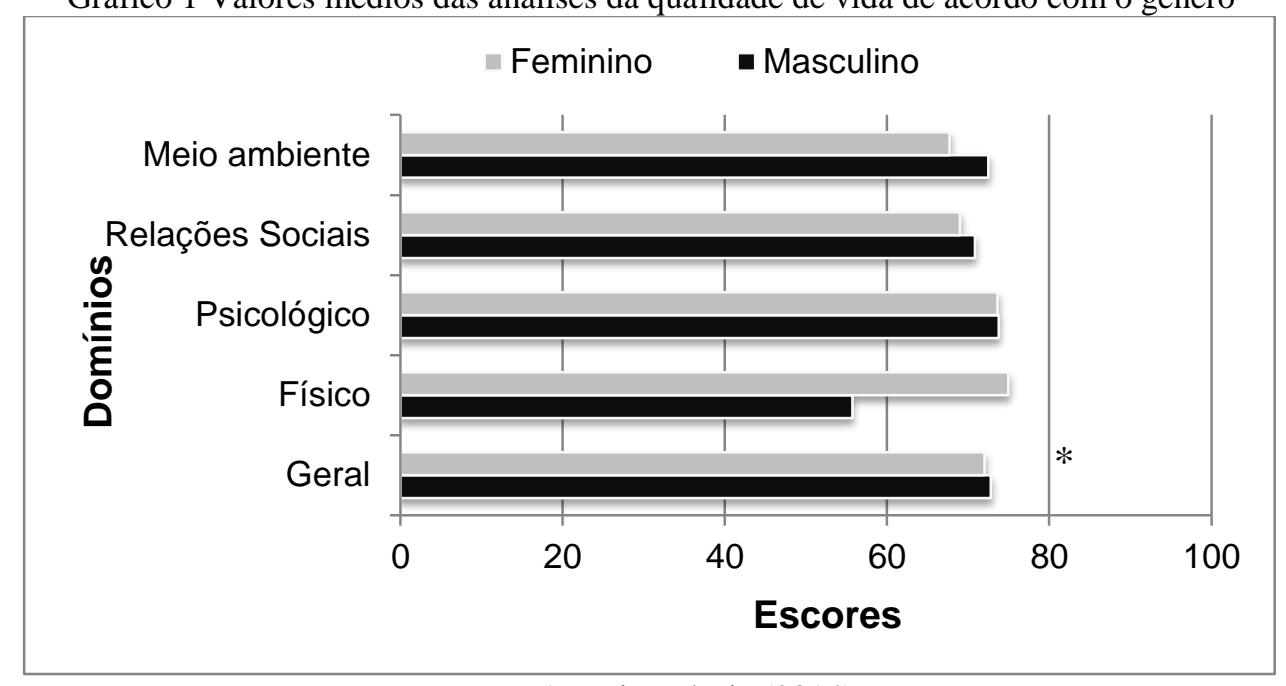

Fonte: Autoria própria (2014).

Já na análise das diferenças entre as modalidades (Gráfico 2), os canoístas mostraram índices significativamente superiores nos domínios físico $(p=0,001)$, psicológico $(p=0,010)$ e relações sociais $(\mathrm{p}=0,012)$. 


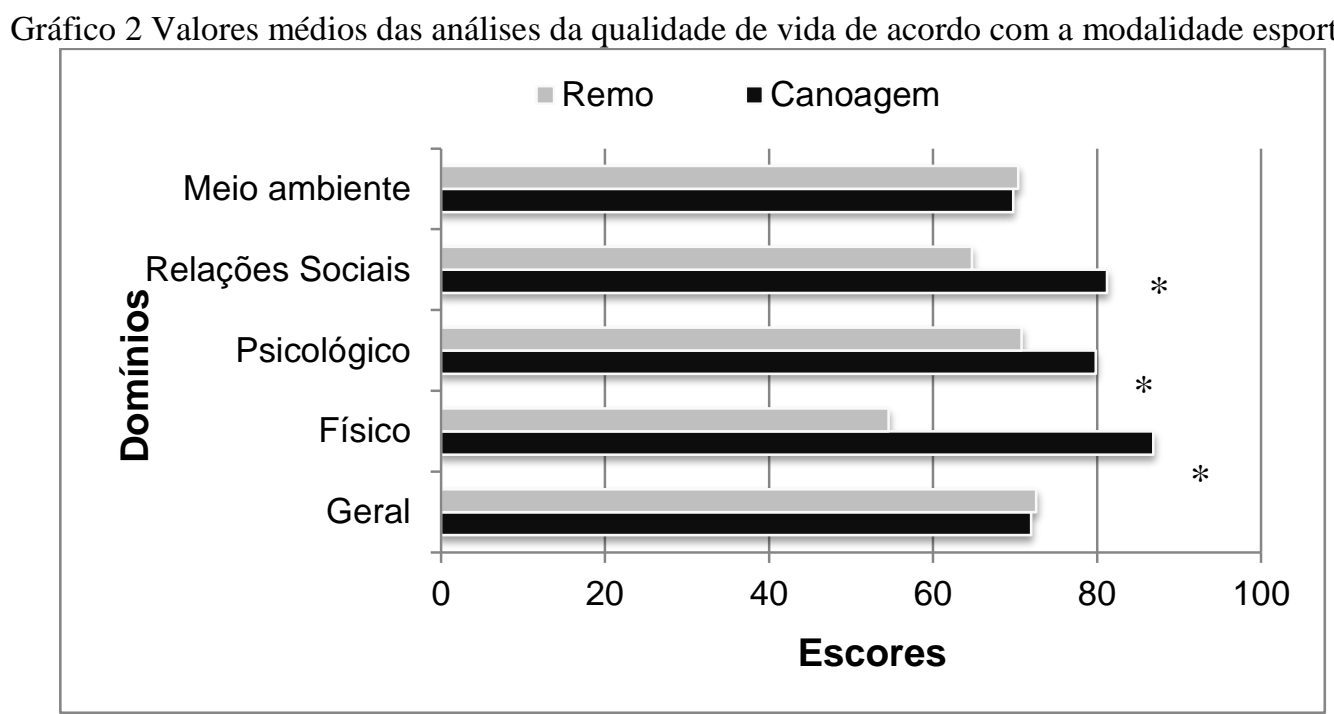

Fonte: Autoria própria (2014).

Os resultados das análises da capacidade explicativa dos domínios para a qualidade de vida geral (Tabela 2) apontaram quatro possíveis modelos de arranjos das variáveis e uma capacidade explicativa de $41,90 \%$ considerando todos os domínios. O domínio meio ambiente foi o que apresentou maior poder preditivo para a qualidade de vida geral com explicação de $24,50 \%$ da variação dos escores.

Tabela 2 - Análises de regressão linear utilizando-se a percepção de qualidade de vida geral como variável dependente

\begin{tabular}{llcc}
\hline \multirow{2}{*}{ Domínios } & \multicolumn{3}{c}{ Qualidade de vida geral } \\
\cline { 2 - 4 } & Constante & 0,054 & \\
\multirow{4}{*}{ Modelo 1 } & Meio ambiente & 0,106 & \\
& Físico & 0,146 & 41,90 \\
& Psicológico & 0,093 & \\
& Relações sociais & 0,621 & \\
\hline \multirow{4}{*}{ Modelo 2 } & Constante & 0,026 & \\
& Meio ambiente & 0,100 & \multirow{2}{*}{40,80} \\
& Físico & 0,145 & \\
& Psicológico & 0,072 & \\
\multirow{4}{*}{ Modelo 3 3 Constante } & 0,014 & \\
& Meio ambiente & 0,103 & 31,50 \\
& Psicológico & 0,219 & \\
\hline \multirow{2}{*}{ Modelo 4 } & Constante & $<0,001$ & \multirow{2}{*}{24,50} \\
& Meio ambiente & 0,031 & \\
\hline \multicolumn{4}{c}{ Fonte: Autoria própria (2014). } \\
\end{tabular}

Com a análise das facetas da avaliação da qualidade de vida com menores e maiores médias (Tabela 3) foram encontradas diferenças na percepção de importância das dimensões comparando os brasileiros com os estrangeiros, enquanto que os dados dos atletas norte-americanos e canadenses apresentaram um comportamento semelhante. De forma geral, as questões com menores médias para os brasileiros estão relacionadas ao domínio meio ambiente e incluem oportunidades de lazer e de recursos financeiros. Já para os atletas estrangeiros, as questões relacionadas ao domínio físico como dor e desconforto e dependência de tratamento médico mostraram-se com resultados mais negativos. As questões gerais de percepção de qualidade de vida e saúde apresentaram uma tendência de escores superiores para os atletas de todas as nacionalidades.

Os atletas norte-americanos e canadenses apresentaram percepção mais positiva de segurança física e proteção superior aos brasileiros. Da mesma forma, os atletas norte-americanos parecem possuir maiores oportunidades de informação. Por outro lado, as questões de autoestima e 
ambiente no lar, que foram apontadas como as mais positivas pelos brasileiros, não foram as principais questões apontadas pelos atletas estrangeiros conforme apresentado na Tabela 3:

Tabela 3 Questões com menores e maiores médias para cada nacionalidade

\begin{tabular}{|c|c|c|c|c|}
\hline Pais & \multicolumn{4}{|c|}{ Questões } \\
\hline \multirow{5}{*}{ Brasil } & Menores médias & $\mathrm{x}(\mathrm{s})$ & Maiores médias & $\mathrm{x}(\mathrm{s})$ \\
\hline & $\begin{array}{l}\text { Participação e oportunidades de } \\
\text { lazer (meio ambiente) }\end{array}$ & $2,83(0,40)$ & Mobilidade (físico) & 5,00 \\
\hline & $\begin{array}{l}\text { Recursos financeiros (meio } \\
\text { ambiente) }\end{array}$ & $3,16(0,98)$ & Autoestima (psicológico) & $4,83(0,40)$ \\
\hline & $\begin{array}{l}\text { Atividade sexual (relações } \\
\text { sociais) }\end{array}$ & $3,60(0,89)$ & Ambiente no lar (meio ambiente) & $4,66(0,51)$ \\
\hline & $\begin{array}{l}\text { Ambiente físico saudável e } \\
\text { oportunidade de adquirir } \\
\text { informação (meio ambiente) }\end{array}$ & $3,66(0,81)$ & Percepção geral de qualidade de vida & $4,66(0,51)$ \\
\hline \multirow[b]{2}{*}{ Canadá } & Dor e desconforto (físico) & 1,00 & Segurança física e proteção (meio ambiente) & 5,00 \\
\hline & $\begin{array}{l}\text { Dependência de tratamento } \\
\text { médico (físico) } \\
\text { Participação e oportunidade de } \\
\text { lazer (meio ambiente) e } \\
\text { sentimentos negativos } \\
\text { (psicológico) }\end{array}$ & $2,25(0,50)$ & $\begin{array}{l}\text { Disponibilidade de cuidados de saúde e sociais } \\
\text { (meio ambiente), Energia e fadiga (domínio } \\
\text { físico), percepção geral de saúde e de qualidade } \\
\text { de vida }\end{array}$ & $4,50(0,58)$ \\
\hline \multirow{4}{*}{ EUA } & Dor e desconforto (físico) & $1,22(0,44)$ & \multirow{2}{*}{$\begin{array}{l}\text { Percepção geral de saúde } \\
\text { Segurança física e proteção (meio ambiente) e } \\
\text { mobilidade (físico) }\end{array}$} & $4,66(0,50)$ \\
\hline & $\begin{array}{l}\text { Dependência de tratamento } \\
\text { médico (físico) }\end{array}$ & $1,33(0,50)$ & & $4,55(0,72)$ \\
\hline & $\begin{array}{l}\text { Sentimentos negativos } \\
\text { (psicológico) }\end{array}$ & $2,22(0,44)$ & \multirow{2}{*}{$\begin{array}{l}\text { Oportunidades de adquirir informação (meio } \\
\text { ambiente), imagem corporal e aparência } \\
\text { (psicológico) e percepção geral de qualidade de } \\
\text { vida }\end{array}$} & \multirow{2}{*}{$4,44(0,52)$} \\
\hline & $\begin{array}{l}\text { Participação e oportunidades de } \\
\text { lazer (meio ambiente) }\end{array}$ & $2,56(0,52)$ & & \\
\hline
\end{tabular}

Fonte: Autoria própria (2014).

\section{Discussão}

Este estudo buscou comparar a percepção de qualidade de vida de um grupo de atletas de canoagem e remo participantes dos jogos Pan-americanos de 2007, de três nacionalidades, por meio do Whoqol-bref. As maiores limitações apontadas se referem ao número baixo de indivíduos da amostra e à falta de equidade entre gêneros nos grupos de análise. Apesar disso, o estudo mostra aspectos pouco explorados pela literatura devido, entre outros fatores, às dificuldades de obtenção de dados de atletas de diferentes nacionalidades.

Resultados de investigações com aplicação do Whoqol-bref em atletas de elite são restritos na literatura especializada. Apesar disso, maiores escores no domínio físico e menores escores no domínio meio ambiente também foram observados no estudo de validação do instrumento para o Brasil (FLECK et al., 2000). O domínio físico, que conta com questões relacionadas à dor e desconforto, energia e fadiga, sono e repouso, mobilidade, atividades da vida cotidiana, dependência de medicação e tratamentos e capacidades para o trabalho foi o que apresentou maiores diferenças entre os atletas brasileiros e estrangeiros e pode indicar um treinamento físico mais exaustivo para os atletas estrangeiros que leve à necessidade de tratamento médico e maiores prevalências de queixas musculoesqueléticas.

Embora tenha sido identificada diferença significativa entre homens e mulheres nos escores do domínio físico, acredita-se que a nacionalidade seja o principal fator associado, já que os grupos canadenses e norte-americanos foram formados, por mulheres e homens, respectivamente. Esse resultado pode apontar para uma semelhança de treinamentos e necessidades físicas para os atletas estrangeiros, bem como, uma especificidade dos atletas brasileiros em termos de qualidade de vida física. É importante apontar que, especialmente em se tratando de atletas, o físico influencia de 
forma importante no domínio psicológico e na qualidade de vida já que, como verificado por Kenttã, Hassmén e Raglin (2001), Meller et al. (2007) e Hutchison et al. (2009), os atletas apresentam importante resposta emocional às lesões, bem como, ao relacionamento com o treinamento, modificando os indicadores de qualidade de vida, tensão, depressão, raiva, vigor e fadiga, podendo levar a situações de overtraining e Síndrome de Burnout.

Em relação aos domínios psicológico e relações sociais, os brasileiros se assemelharam mais aos canadenses do que aos norte-americanos. Os atletas norte-americanos apresentaram piores escores no domínio psicológico. Dados sobre a saúde mental dos atletas de elite, também, são restritos na literatura devido a uma maior atenção aos problemas físicos. Pesquisas têm mostrado que os atletas formam uma população com alto risco psicológico e para doenças psiquiátricas, como o abuso de substâncias e distúrbios alimentares, sendo que esses problemas podem não ser percebidos pelos treinadores e talvez agravados pela pressão dos resultados (LINDER et al., 1991; RESCH; HAÁSZ, 2009; GLICK; HORSFALL, 2005; ELLENDER; LINDER, 2005). De forma geral, os dados indicam que os atletas brasileiros possuem relações sociais e qualidade de vida psicológica mais favoráveis que os atletas estrangeiros.

A percepção geral de qualidade de vida foi semelhante para as diferentes nacionalidades e os domínios apresentaram uma capacidade explicativa de $41,90 \%$ para a percepção geral de qualidade de vida, resultados similares ao estudo de validação do instrumento para o Brasil (FLECK et al., 2000). De forma mais específica, o domínio meio ambiente, que engloba indicadores como segurança, clima, transportes, oportunidades de adquirir novos conhecimentos e de lazer e recursos financeiros, apresentou maior capacidade individual para explicação $(24,50 \%)$ e, junto com os indicadores do domínio físico, devem ser priorizados nas medidas de intervenção para promoção da qualidade de vida de atletas de canoagem e remo.

As análises das facetas dos domínios do Whoqol-bref com menores e maiores médias de pontuação colaboraram para uma melhor visualização das causas dos resultados gerais. Nesta análise, evidenciou-se clara diferença entre os atletas brasileiros e estrangeiros. Em atletas estrangeiros, as facetas do domínio físico como dor, desconforto e dependência de tratamento médico apresentaram piores escores corroborando com o estudo de Mcallister et al. (2001) em amostra de 562 atletas colegiais de elite norte-americanos, no qual foi verificado que as variáveis relacionadas ao domínio físico, especialmente as lesões e dores, foram os principais fatores associados à qualidade de vida relacionada à saúde avaliando-se pelo SF-36.

Os atletas norte-americanos e canadenses apresentaram percepção mais positiva de segurança física e proteção e de oportunidades de informação quando comparados aos brasileiros. Este fato pode estar associado ao grau de desenvolvimento econômico dos países. Já as questões de autoestima e ambiente no lar, que foram apontadas como as mais positivas pelos brasileiros, não foram as principais questões apontadas pelos atletas estrangeiros e podem estar associadas ao ambiente em que estava sendo realizada a competição, resultados que precisam ser confirmados em futuros estudos. Ambiente e políticas públicas, personalidade, liberdade, democracia, família, cultura, locais para lazer, dentro outros completam uma rede de fatores que influenciam na forma com que as pessoas vivem e na sua qualidade de vida.

De forma geral, os resultados encontrados fornecem evidências para alguns constructos teóricos sobre qualidade de vida como o de Minayo, Hartz e Buss (2000), ao apontar a necessidade de relatividade da noção de qualidade de vida ao plano individual, considerando tanto referências:

a) históricas, já que o desenvolvimento econômico, social e tecnológico traz às sociedades parâmetros de qualidade de vida diferenciados, na mesma sociedade, em outra etapa histórica;

b) culturais, já que diferentes povos apresentam valores e necessidades diversos;

c) estratificações ou classes sociais, pois normalmente os parâmetros de percepção de qualidade de vida e bem-estar se voltam aos padrões das camadas superiores, especialmente em países com grandes desigualdades sociais. 


\section{Considerações finais}

Considerando os escores dos domínios de qualidade de vida avaliados, as maiores necessidades dos atletas norte-americanos estão associadas aos domínios relações sociais e físico, dos canadenses ao domínio físico e dos brasileiros ao domínio meio ambiente evidenciando diferenças marcantes entre as diferentes nacionalidades.

\section{Referências}

CASPERSEN, C. J.; POWELL, K. E.; CHRISTENSON, G. M. Physical activity, exercise, and physical fitness: definitions and distinctions for health-related research. Public Health Reports, v. 100, p. 126-131, Mar./Apr. 1985.

ELLENDER, L.; LINDER, M. M. Sports pharmacology and ergogenic aids. Primary Care, Philadelphia, v. 32, n. 1, p. 277-292, Mar. 2005. crossef

FERREIRA, R. M.; MORAES, L.C. Influencia da família na primeira fase de desenvolvimento da carreira de nadadores medalhistas olímpicos brasileiros. Motriz, Rio Claro, v. 8, n. 2, p. 42-51, 2012.

FLECK, M. P. A.; LOUZADA, S.; MARTA XAVIER, M.; CHACHAMOVICH, E.; VIEIRA, G.; SANTOS, L.; PINZON, E. V. Aplicação da versão em português do instrumento abreviado da qualidade de vida WHOQOL-bref. Revista de Saúde Pública, Rio de Janeiro, v. 34, n. 2, p. 178183, abr. 2000.

GLICK, I. D.; HORSFALL, J. L. Diagnosis and psychiatric treatment of athletes. Clinics in Sports Medicine, Philadelphia, v. 24, n. 4, p. 771-781, Oct. 2005. Crossef

HENSEL, P.; PERRONI, M. G.; LEAL JUNIOR, E. C. P. Lesões musculoesqueléticas na temporada de 2006 em atletas da seleção brasileira feminina principal de canoagem velocidade. Acta Ortopédica Brasileira, São Paulo, v. 16, n. 4, p. 233-237, dez. 2008. Cross'ef

HUFFMAN E. A.; YARD, E. E.; FIELDS, S. K.; COLlINS, C. L.; COMSTOCK, R. L. Epidemiology of rare injuries and conditions among United States high school athletes during the 2005-2006 and 2006-2007 school years. Journal of Athletic Training, Dallas, v. 43, n. 6, p. 624630, Oct./Dec. 2008. rossef

HUTCHISON, M.; MAINWARING, L. M.; COMPER, P.; RICHARDS, D. W.; BISSCHOP, S. M. Differential emotional responses of varsity athletes to concussion and musculoskeletal injuries. Clinical Journal of Sport Medicine, New York, v. 19, n. 1, p. 13-19, Jan. 2009. Crossef

KENTTÄ, G.; HASSMÉN, P.; RAGLIN, J. S. Training practices and overtraining syndrome in Swedish age-group athletes. International Journal of Sports Medicine, Stuttgart, v. 22, n. 6, p. 460-465, Aug. 2001. rossef

KNOWLES, S. B.; MARSHALL, S. W.; GUSKIEWICZ, K. M. Issues in estimating risks and rates in sports injury research. Journal of Athletic Training, Dallas, v. 41, n. 2, p. 207-215, 2006.

LEMOS, L. F. C.; TEIXEIRA, C. S.; MOTA, C. B. Lombalgia e o equilíbrio corporal de atletas da seleção brasileira feminina de canoagem velocidade. Revista Brasileira de Cineantropometria \& Desempenho Humano, v. 12, n. 6, p. 457-463, 2010. Crossef 
LINDER, D. E.; BREWER, B. W.; VAN RAALTE, J. L.; DELANGE, N. A negative halo for athletes who consult sport psychologists: replication and extension. Journal of Sport \& Exercise Psychology, Champaign, v. 13, n. 2, p. 133-148, June 1991.

MARWAN, Y.; BEHBEHANI, A.; AL-MOUSAWI, A.; MULLA-JUMA, A.; SADEQ, H.; SHAH, N. Sports injuries among professional male athletes in Kuwait: prevalence and associated factors. Medical Principles and Practice, New York, v. 21, n. 2, p. 171-177, Oct. 2011. Crossef

MCALliSTER, D. R.; MOTAMEDI, A. R.; HAME, S. L.; SHAPIRO, M. S.; DOREY, F. J. Quality of Life Assessment in Elite Collegiate Athletes. The American Journal of Sports Medicine, Baltimore, v. 29, n. 6, p. 806-810, Nov./Dec. 2001.

MELLER, R.; KRETTEK, C.; GÖSLING, T.; WÄHLING, K.; JAGODZINSKI, M.; ZEICHEN, J. Recurrent shoulder instability among athletes: changes in quality of life, sports activity, and muscle function following open repair. Knee Surgery, Sports Traumatology, Arthroscopy, Heidelberg, v. 15, n. 3, p. 295-304, 2007.

MINAYO, M. C. S.; HARTZ, Z. M. A.; BUSS, P. M. Qualidade de vida e saúde: um debate necessário. Ciência \& Saúde Coletiva, Rio de Janeiro, v. 5, n. 1, p. 7-18, 2000.

RECHEL, J. A.; YARD, E. E.; COMSTOCK, R. D. An epidemiologic comparison of high school sports injuries sustained in practice and competition. Journal of Athletic Training, Dallas, v. 43, n. 2, p. 197-204, Apr./June 2008. crossef

RENWICK, R.; BROWN, I. The center for health promotion's conceptual approach to quality of life. In: RENWICK, R.; BROWN, I.; NAGLER, M. (Ed.). Quality of life in health promotion and rehabilitation: conceptual approaches, issues and applications. Thousand Oaks: Sage, 1996.

RESCH, M.; HAÁSZ, P. The first epidemiology survey among Hungarian elite athletes: eating disorders, depression and risk factors. Orvosi Hetilap, Budapest, v. 150, n. 1, p. 35-40, Jan. 2009. crossef

SILVA, C. C.; WOFF, M.; DECHECHI, C. J.; ALMEIDA, A. G.; NAKAMURA, F. Y. Análise da cinética de remoção de lactato em atletas de canoagem slalom. Revista Brasileira de Ciências do Esporte, v. 35, n. 2, p. 425-439, 2013. Crossef

WALLS, R. J.; BRENNAN, S. A.; HODNETT, P.; O'BYRNE， J. M.; EUSTACE, S. J.; STEPHENS, M. M. Overuse ankle injuries in professional Irish dancers. Journal of Foot and Ankle Surgery, Paris, v. 16, n. 1, p. 45-49, Mar. 2010. rrossef

WARBURTON, D. E. R.; NICOL, C. W.; BREDIN, S. S. D. Health benefits of physical activity: the evidence. CMAJ, v. 174, n. 6, p. 801-809, 2006. erossef

WHOQOL GROUP. The development of the World Health Organization quality of life assessment instrument (the WHOQOL). In: ORLEY, J.; KUYKEN, W. (Ed.). Quality of life assessment: international perspectives. Heidelberg: Springer, 1994. rossef 